\title{
Standard anti-tuberculosis treatment and hepatotoxicity: do dosing schedules matter?
}

\author{
K.C. Chang*, C.C. Leung*, W.W. Yew" and C.M. Tam*
}

\begin{abstract}
A nested case-control study was conducted in order to examine whether dosing schedules of standard pyrazinamide-containing anti-tuberculosis (TB) treatment (standard treatment) might affect hepatotoxicity.
\end{abstract}

The present authors retrospectively identified all patients with hepatitis using biochemical criteria from a cohort of 3,007 clinic patients who commenced anti-TB treatment from January 1 to June 30, 2001. Each case with hepatitis between 1-9 weeks post-TB treatment was compared using conditional logistic regression analysis with two controls selected randomly from patients without hepatitis in the same period and matched by sex, age and standard treatment. Impacts of sex and age were examined by logistic regression analysis of cases and patients without hepatitis.

Hepatitis occurred in 167 patients, of whom 96 qualified as cases. A conditional logistic risk model identified hepatitis B surface antigen carriage as the only risk factor (odds ratio (95\% confidence interval $(\mathrm{Cl})) 1.8(1.1-3.1))$. Logistic regression analysis showed that sex was nonsignificant but ageing increased the odds of hepatitis. The risk of hepatitis increased from $2.6 \%(1.9-3.5 \%)$ to $4.1 \%(3.2-5.3 \%)$ as age exceeded 49 yrs.

Dosing schedules in the first 9 weeks have little impact on hepatotoxicity. If patients at risk of both hepatitis and relapse receive standard treatment, daily dosing is preferable.

KEYWORDS: Dosing schedules, hepatitis, hepatotoxicity, risk factors, treatment, tuberculosis

$\mathbf{R}$ isk factors for hepatitis have been evaluated by a number of observational studies [1-15] but few addressed treatment-related factors [12-15]. While the latter focused on the risk of hepatitis induced by individual drugs, the impact of dosing schedules has not been adequately addressed.

Whether dosing schedules affect the risk of druginduced hepatitis may bear clinical relevance. A nested case-control study showed that standard thrice-weekly anti-tuberculosis (TB) treatment increased the risk of relapse in comparison with daily treatment, and the risk difference might be clinically significant for patients with cavitary TB [16]. Three randomised controlled trials involving combination therapy with isoniazid, rifampicin and pyrazinamide in the initial phase showed that daily treatment may be more hepatotoxic than thrice-weekly treatment [1719]. However, the findings from these three trials may not be applicable to the standard 6-month regimen for two reasons. First, pyrazinamide was administered for 3-6 months in two trials $[17,18]$. The hepatotoxicity of daily and thrice-weekly treatment might be similar if pyrazinamide was administered for $\sim 2$ months. Second, in contrast to the current practice, the dosages of rifampicin and pyrazinamide for daily and thrice-weekly treatment in the remaining clinical trial were identical [19]. Thus, any significant difference between daily and thrice-weekly regimens may be inapplicable to usual settings. If the standard pyrazinamide-containing initial phase is significantly more hepatotoxic than the thrice-weekly counterpart, it may be necessary to balance benefit against risk in determining the optimal initial phase regimen for subgroups at risk of both relapse and hepatitis.

Given the paucity of studies on dosing schedules and hepatotoxicity, and the relatively low prevalence of drug-induced hepatitis, a nested casecontrol study was conducted in order to examine whether dosing schedules of standard pyrazinamide-containing anti-TB treatment might affect hepatotoxicity in the first 9 weeks.

\section{MATERIAL AND METHODS}

A nested case-control study was conducted in order to examine the impact of dosing schedules on hepatotoxicity. From a computerised registry of TB patients treated in government chest clinics, the present authors retrospectively identified all patients with hepatitis after TB treatment from a cohort who commenced treatment from January
AFFILIATIONS

*Tuberculosis and Chest Service, Centre for Health Protection, Department of Health, and "Tuberculosis and Chest Unit, Grantham Hospital, Hong Kong, China.

CORRESPONDENCE

K.C. Chang

Yaumatei Chest Clinic 2nd floor Yaumatei Jockey Club Polyclinic 145 Battery Street

Kowloon

Hong Kong

China

Fax: 85223743575

E-mail: kc chang@dh.gov.hk

Received:

July 092006

Accepted after revision:

August 282006

SUPPORT STATEMENT

No financial support was received for this study and all authors declare they have no competing interests.

STATEMENT OF INTEREST None declared. 
1 to June 30,2001 . Hepatitis was defined as elevation of alanine aminotransferase (ALT) or total bilirubin above thrice and twice their upper limits of normal (ULN), respectively. This condition was presumed to be drug-induced unless proven otherwise. Anti-TB treatment was given largely under direct observation either at the hospital or in any of the 18 government chest clinics (12 full-time and six part-time) distributed in different districts of Hong Kong (China) within the range of the Tuberculosis and Chest Service. Trained healthcare workers watched out for treatment-related adverse events and took immediate defaulter-tracing actions for nonadherent patients.

The present nested case-control study was confined to the first 9 weeks after TB treatment. Patients with hepatitis $<1$ week post-treatment were excluded, as it would probably take $\geqslant 1$ week to show any difference between daily and thriceweekly treatment. Each case was matched by sex, age (within 3 yrs of difference) and treatment, with two controls selected randomly from patients without hepatitis during the first 9 weeks. Cases and matched controls received standard pyrazinamide-containing treatment for $\geqslant 70 \%$ of the time before the diagnosis of hepatitis and the end of the first 9 weeks, respectively. The first two eligible controls were selected. Cases and matched controls were compared using conditional logistic regression analysis. Impacts of sex and age on hepatotoxicity were examined by logistic regression analysis of cases and all patients without hepatitis in the first 9 weeks. Medical records of all cases and controls were retrieved for verification and data capture.

Table 1 shows the list of predictor variables analysed by univariate conditional logistic regression analysis. Dosing schedules and predictor variables with $\mathrm{p}$-values $\leqslant 0.2$ by univariate analysis were forced into a conditional logistic risk model to evaluate the impact of dosing schedules on drug-induced hepatitis.

Approval was obtained from the institutional review board for conducting the study. Patient consent was deemed unnecessary due to the observational nature of the present study.

\section{RESULTS}

The present cohort comprised 3,007 patients who commenced TB treatment from January 1 to June 30, 2001 and received treatment partly or entirely from government chest clinics. The majority were Chinese (97\%) and permanent residents of Hong Kong (96\%). A total of 192 episodes of drug-induced hepatitis occurred in 167 patients $(5.6 \%)$, of whom $123(4.1 \%)$ cases were diagnosed within the first 9 weeks. The frequency distribution of drug-induced hepatitis by age group was as follows: $\leqslant 49 \mathrm{yrs}, 64$ (38.3\%); 50-64 yrs, 41 (24.6\%); and >64 yrs, 62 (37.1\%). Four patients, all aged $>64$ yrs, died after developing hepatitis (table 2). The case-fatality rate of drug-induced hepatitis was $6.5 \%$ for the age group $>64$ yrs. The distribution of the first episode of drug-induced hepatitis by the time of diagnosis was skewed, with mode at 1.1 week, median at 5 weeks, interquartile range $2.4-8.3$ weeks and range $0.3-$ 44.4 weeks. The risk of hepatitis (95\% confidence interval (CI)) in the first 9 weeks of TB treatment relative to the remaining period was 2.7 (1.9-3.8).
Out of 123 patients who had hepatitis within the first 9 weeks, a total of 27 were excluded from the present nested casecontrol analysis for the following reasons: two for overdose (one received 3,500 $\mathrm{mg}$ pyrazinamide daily; the other received $1,000 \mathrm{mg}$ isoniazid and 2,500 $\mathrm{mg}$ pyrazinamide daily), five for onset of hepatitis in $<1$ week and 20 for ineligible treatment (either non-pyrazinamide regimens or standard pyrazinamidecontaining regimens given for $<70 \%$ of the time before hepatitis). Thus, 96 cases were eligible, comprising 31 females and 65 males. A total of $59(61.5 \%)$ were aged $>49$ yrs (table 1 ). ALT was $3-5$ times its ULN in $45.8 \%$ of the cases, $>10$ times its ULN in $25.0 \%$ and $<3$ times its ULN in $5.2 \%$ (increase in total bilirubin met the criterion for biochemical hepatitis in these cases). The proportion of severe hepatitis (ALT $\geqslant 10$ times its ULN) was higher among patients who started treatment in the chest clinic than those who started treatment in the hospital (33 versus $18 \%$ ), but the difference did not reach statistical significance $(p=0.09)$. Cases with ALT levels $\geqslant 5$ times its ULN (moderate or severe hepatitis) were significantly more likely to have symptoms of hepatitis than those with lower ALT levels ( 79.5 versus $54.5 \%$; $p=0.02$; missing data $\sim 25 \%$ ). No significant association was found between hepatitis B surface antigen (HBsAg) carriers and moderate or severe hepatitis.

A total of 96 cases were compared with 192 matched controls in a conditional logistic risk model. Approximately $85 \%$ of the cases and $95 \%$ of the matched controls received standard pyrazinamide-containing treatment for $\geqslant 90 \%$ of the time before the diagnosis of hepatitis and the end of the first 9 weeks, respectively. Table 1 shows the results of univariate conditional logistic regression analysis. Table 3 shows the results of multiple conditional logistic regression analysis in two models. Model A excluded drinking history and HBsAg status from the analysis. Model B included drinking history and HBsAg status. It was assumed that all patients with unknown HBsAg status and unknown drinking history were HBsAg negative and nondrinkers or social drinkers, respectively. Model B identified only one risk factor for drug-induced hepatitis: HBsAg carriers (odds ratio (95\% confidence interval) 1.8 (1.1-3.1)). Both models demonstrated no significant association between dosing schedules and drug-induced hepatitis (1.1 (0.6-1.9)). No significant association was found between dosing schedules and the time of onset of hepatitis or the severity of hepatitis according to ALT levels.

Logistic regression analysis of 96 cases and 2,789 patients in the cohort with no hepatitis in the first 9 weeks showed that sex had a nonsignificant impact, but age $>49$ yrs increased the odds of hepatitis $(1.6(1.1-2.5))$. The risk of hepatitis in the first 9 weeks increased from $2.6 \%(1.9-3.5 \%)$ to $4.1 \%(3.2-5.3 \%)$ where age was $>49$ yrs. The corresponding risk for females increased from $2.3 \%(1.4-3.7 \%)$ to $4.7 \%(2.9-7.5 \%)$ and from $2.8 \%(1.9-4.2 \%)$ to $3.9 \%(2.9-5.3 \%)$ for males.

\section{DISCUSSION}

The present authors have demonstrated using a conditional logistic risk model that dosing schedules of standard pyrazinamide-containing treatment in the first 9 weeks have no significant impact on drug-induced hepatitis. The same conclusion is reached regardless of the inclusion of $\mathrm{HBsAg}$ status or drinking history. As it has been shown that daily treatment in comparison with thrice-weekly treatment 


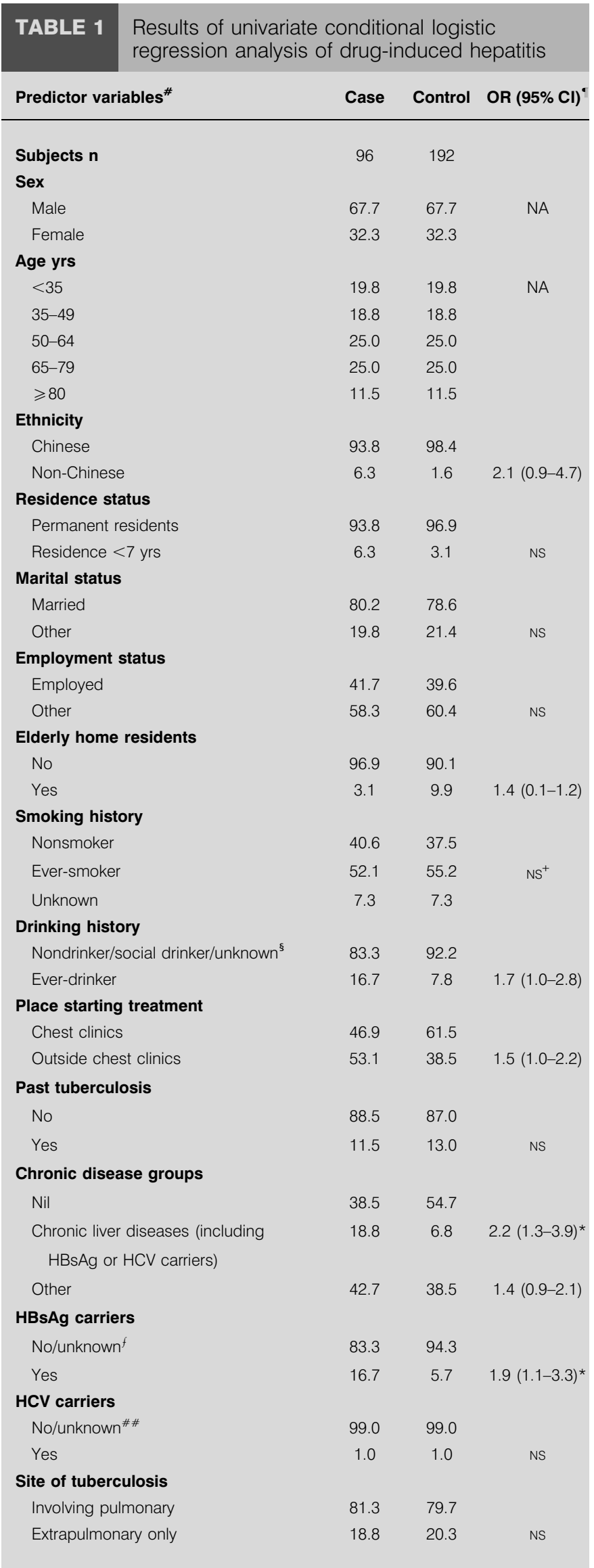

\section{TABLE 1 continued}

\begin{tabular}{|c|c|c|c|}
\hline Predictor variables $^{\#}$ & Case & Control & OR $(95 \% \mathrm{Cl})^{\circ}$ \\
\hline \multicolumn{4}{|l|}{ Extent of pulmonary disease } \\
\hline$<$ right lung & 82.1 & 84.9 & \\
\hline$>$ right lung & 14.1 & 11.8 & $\mathrm{NS}^{+}$ \\
\hline Unknown & 3.8 & 3.3 & \\
\hline \multicolumn{4}{|l|}{ DOT at chest clinic } \\
\hline$<75 \%$ & 56.3 & 49.0 & \\
\hline $75-100 \%$ & 43.8 & 51.0 & NS \\
\hline \multicolumn{4}{|l|}{ Dosing schedules" } \\
\hline Thrice-weekly & 20.8 & 27.6 & \\
\hline Daily/Mixed ${ }^{++}$ & 79.2 & 72.4 & NS \\
\hline \multicolumn{4}{|l|}{ Dosing schedules" } \\
\hline Thrice-weekly & 20.8 & 27.6 & \\
\hline Daily/Mixed ${ }^{++} Z<30 \mathrm{mg} \cdot \mathrm{kg}^{-1} \cdot$ day $^{-1}$ & 24.0 & 24.5 & \\
\hline Daily $/$ Mixed $^{++} Z=30-35 \mathrm{mg} \cdot \mathrm{kg}^{-1} \cdot$ day $^{-1}$ & 27.1 & 25.0 & \\
\hline Daily/Mixed ${ }^{++} Z \geqslant 35 \mathrm{mg} \cdot \mathrm{kg}^{-1} \cdot$ day $^{-1}$ & 26.1 & 22.4 & $\mathrm{NS}^{+}$ \\
\hline Daily/Mixed ${ }^{++} Z=$ unknown & 2.0 & 0.5 & \\
\hline
\end{tabular}

Data are presented as \%, unless otherwise stated. OR: odds ratio; $\mathrm{Cl}$ confidence interval; NA: not applicable due to matching; Ns: nonsignificant with p>0.2; HBsAg: hepatitis B surface antigen; HCV: hepatitis C; DOT: directly observed treatment; Z: pyrazinamide. ": first subgroup is reference. ": numerical values shown only when $p$-values of main entry $\leqslant 0.2{ }^{+}$: excluding unknown from analysis. ${ }^{5}$ : $83.3 \%$ of cases and $92.2 \%$ of controls had unknown drinking history. ${ }^{f}: 17.7 \%$ of cases and $73.4 \%$ of controls had unknown HBsAg status. \#\#: $99 \%$ of both cases and controls had unknown HCV status. "\%: dosing schedules would be regarded as daily or thrice-weekly when either was $>90 \%{ }^{++}$: mixed schedules referred to the coexistence of thrice-weekly and daily regimens when both were $<90 \% ; 10.4 \%$ of cases and $7.3 \%$ of controls received a mixed dosing schedule. ${ }^{*}: p \leqslant 0.05$.

significantly reduces the risk of relapse among patients with cavitary TB [16], daily dosing is preferable if patients at risk of both hepatitis and relapse receive standard pyrazinamidecontaining anti-TB regimens.

Although three randomised controlled trials [17-19] showed that daily treatment in comparison with thrice-weekly treatment significantly increased the risk of "hepatic reactions" or "hepatic adverse events", in none of these clinical trials were the latter terms, which were not the primary study end-points, well defined. Hepatic reactions were vaguely defined as abnormalities in liver function tests, leading to a modification to the regimen [17] or implicitly described as including symptomless rises in serum ALT and clinically evident hepatitis [18]. Hepatic adverse events were indirectly described as events resulting in a change of treatment or an interruption of $\geqslant 7$ days [19]. Where analysis was confined to patients with jaundice (all occurring within the first 2 months) [17], there was no significant difference between daily and thrice-weekly regimens. It may be argued that the negative finding in the present study is a type II error. However, the current nested case-control study based on a cohort of 3,007 patients should outstrip any of the three controlled trials by both the number of patients and its statistical power. 
TABLE 2 Clinical profiles of four patients who died after developing drug-induced hepatitis

\begin{tabular}{lccccccccc} 
Sex & Age yrs & $\begin{array}{c}\text { Onset of } \\
\text { hepatitis weeks }\end{array}$ & HBsAg & HCVAb & Drinking history & $\begin{array}{c}\text { Predominant } \\
\text { hepatotoxic drugs }\end{array}$ & Symptoms $^{*}$ & ALT $^{*} \mathbf{U}^{-L^{-1}}$ & Bilirulin $^{\boldsymbol{*}} \boldsymbol{\mu m o l \cdot \mathbf { L } ^ { - 1 }}$ \\
\hline Male $^{+}$ & 69 & 6 & + & NA & NA & H, R, Z & NA & 149 \\
Female & 91 & 8.4 & NA & NA & NA & H, R & NA & 27 & Normal \\
Male & 67 & 17.3 & + & NA & NA & H, R, Z & Tiredness & 761 & 73 \\
Male & 75 & 27 & NA & NA & NA & H, R & No & 750 & 162 \\
\hline
\end{tabular}

HBsAg: hepatitis B surface antigen; HCVAb: hepatitis C antibody; ALT: alanine aminotransferase; NA: not available; H: isoniazid; R: rifampicin; Z: pyrazinamide. ${ }^{\#}$ : within the previous 4 weeks; ${ }^{\top}$ : at the time of diagnosis; ${ }^{+}$: overdosed with pyrazinamide $3,500 \mathrm{mg} \cdot$ day $^{-1}$.

Although controlled trials suggested that the addition of pyrazinamide to regimens containing rifampicin and isoniazid did not increase the risk of hepatitis [20,21], the present study showed that the risk of hepatitis by biochemical criteria in the first 9 weeks of TB treatment, when pyrazinamide was frequently added to isoniazid and rifampicin, was $\sim 2.7$ times that of the subsequent period, when pyrazinamide was seldom used $(p<0.001)$. This corroborated the high incidence of pyrazinamide-induced hepatotoxicity (which implied toxicity of pyrazinamide added to isoniazid and rifampicin) relative to that of isoniazid-induced hepatotoxicity found by a cohort study [15]. The negative findings from controlled clinical trial might have been caused by insufficient statistical power or differences from the usual service settings.

The present study reaffirmed a significantly higher risk of drug-induced hepatitis among older patients $[1,2,4,6,7,13$, 15] although a few studies showed negative findings [5, 11]. The present findings also corroborated a case-control study in Hong Kong that showed only age and hepatitis B infection were significantly related to anti-TB treatment [6], although the risk of drug-induced hepatitis due to chronic hepatitis B may have been over-estimated by assuming negative results for those with unknown HBsAg. Two studies showed no significant association between drug-induced hepatitis and HBsAg carriers [4, 10], but one of the studies [10] showed that HBsAg carriers were more likely than non-carriers to develop moderate-to-severe drug-induced hepatitis. This finding was not reproduced in the present study.

The present authors failed to demonstrate a significant association between alcoholism and drug-induced hepatitis, which had been shown by PANDE et al. [1] and Devoto et al. [5]. Two studies also showed negative findings [2, 7]. The negative finding in the present study could have been caused by both selection and misclassification bias. Drinkers with baseline liver function dysfunction might be less likely to receive standard pyrazinamide-containing regimens and thus may have been excluded from the present study. Drinking history was missing from a considerable proportion of patients.

The association between drug-induced hepatitis and sex is controversial. The present study showed no association between sex and drug-induced hepatitis. A few studies showed that female patients were more vulnerable to druginduced hepatitis $[2,3,5,7,15]$, while some showed no sex difference $[4,11,13,22]$.
The association between drug-induced hepatitis and the severity of TB is also contentious. Although a few studies demonstrated a positive finding $[1,2,9]$, the present study and another [4], both among Chinese patients, showed no association.

A higher proportion of patients with severe hepatitis was found among those who started treatment in the clinic than their counterparts in the hospital. This may be attributed to early diagnosis of drug-induced hepatitis in the hospital, achieved by more frequent routine monitoring of liver biochemistry. Although the current study was not specifically designed to address the usefulness of routine monitoring for drug-induced hepatitis, it is noteworthy that one of the four fatalities was asymptomatic despite having ALT $>10$ times its ULN at diagnosis of hepatitis. In view of a case-fatality rate of $6.5 \%$ for the age group $>64 \mathrm{yrs}$, a considerable proportion of asymptomatic hepatitis $(20.5 \%$ of cases with ALT $\geqslant 5$ times its ULN and $45.5 \%$ of cases with ALT $<5$ times its ULN) and the predominance of hepatitis in the first 2 months, it may be prudent to monitor liver biochemistry biweekly during this period for high-risk patients.

In summary, age $>49 \mathrm{yrs}$ and hepatitis B surface antigen carriage increase the risk of drug-induced hepatitis. Dosing schedules of standard pyrazinamide-containing regimens have little impact on hepatotoxicity in the first 9 weeks.

TABLE 3 Multiple conditional logistic regression analysis of drug-induced hepatitis

\begin{tabular}{lcc} 
Predictor variables $^{\#}$ & Model A & Model B $^{+}$ \\
\hline Mixed or daily dosing schedules & $1.1(0.6-1.9)$ & $1.1(0.6-1.9)$ \\
Non-Chinese & $1.9(0.8-4.4)$ & $1.8(0.8-4.1)$ \\
Elderly home residents & $0.3(0.1-1.0)$ & $0.4(0.1-1.2)$ \\
Treatment started in hospital & $1.6(1.0-2.5)$ & $1.5(0.9-2.4)$ \\
HBsAg carriers & NA & $1.8(1.1-3.1)^{\star}$ \\
Ever-drinkers & NA & $1.4(0.8-2.5)$
\end{tabular}

HBsAg: hepatitis B surface antigen; NA: not applicable. ${ }^{*}$ : test for multicollinearity showed that $\mathrm{HBsAg}$ carrier status was significantly correlated with chronic liver disease subgroup under coexisting disease group, which was thus excluded from the conditional logistic risk model; ": excluding ever-drinkers and HBsAg carriers; ${ }^{+}$: including ever-drinkers and HBsAg carriers. ${ }^{*}$ : $p \leqslant 0.05$. 


\section{ACKNOWLEDGEMENTS}

The authors would like to thank all colleagues in the Hong Kong Tuberculosis and Chest Service (Hong Kong, China) for their contribution to the computerised tuberculosis registry. The authors are also indebted to the following doctors who provided missing data: K. S. Chan of Haven of Hope Hospital, Y. C. Chan of Wong Tai Sin Hospital, C. H. Chau of Grantham Hospital, J. C. M. Ho of Queen Mary Hospital, S. S. Ho of Alice Ho Miu Ling Nethersole Hospital, Y. W. Mok of Kowloon Hospital, and W. C. Yu of Princess Margaret Hospital, Hong Kong, China.

\section{REFERENCES}

1 Pande JN, Singh SP, Khilnani GC, Khilnani S, Tandon RK. Risk factors for hepatotoxicity from antituberculosis drugs: a case-control study. Thorax 1996; 51: 132-136.

2 Dossing M, Wilcke JT, Askgaard DS, Nybo B. Liver injury during antituberculosis treatment: an 11-year study. Tuber Lung Dis 1996; 77: 335-340.

3 Ormerod LP, Horsfield N. Frequency and type of reactions to antituberculosis drugs: observations in routine treatment. Tuber Lung Dis 1996; 77: 37-42.

4 Hwang SJ, Wu JC, Lee CN, et al. A prospective clinical study of isoniazid-rifampicin-pyrazinamide-induced liver injury in an area endemic for hepatitis B. J Gastroenterol Hepatol 1997; 12: 87-91.

5 Devoto FM, Gonzalez C, Iannantuono R, Serra HA, Gonzalez CD, Saenz C. Risk factors for hepatotoxicity induced by antituberculosis drugs. Acta Physiol Pharmacol Ther Latinoam 1997; 47: 197-202.

6 Wong WM, Wu PC, Yuen MF, et al. Antituberculosis drugrelated liver dysfunction in chronic hepatitis B infection. Hepatology 2000; 31: 201-206.

7 Teleman MD, Chee CBE, Earnest A, Wang YT. Hepatotoxicity of tuberculosis chemotherapy under general programme conditions in Singapore. Int J Tuberc Lung Dis 2002; 6: 699-705.

8 Fernandez-Villar A, Sopena B, Fernandez-Villar J, et al. The influence of risk factors on the severity of anti-tuberculosis drug-induced hepatotoxicity. Int J Tuberc Lung Dis 2004; 8: 1499-1505.

9 Shakya R, Rao BS, Shrestha B. Incidence of hepatotoxicity due to antitubercular medicines and assessment of risk factors. Ann Pharmacother 2004; 38: 1074-1079.

10 Lee BH, Koh WJ, Choi MS, et al. Inactive hepatitis B surface antigen carrier state and hepatotoxicity during antituberculosis chemotherapy. Chest 2005; 127: 1304-1311.
11 Gulbay BE, Gurkan OU, Yildiz OA, et al. Side effects due to primary antituberculosis drugs during the initial phase of therapy in 1149 hospitalized patients for tuberculosis. Respir Med 2006; 100: 1834-1842.

12 Singh J, Arora A, Garg PK, Thakur VS, Pande JN, Tandon RK. Antituberculosis treatment-induced hepatotoxicity: role of predictive factors. Postgrad Med J 1995; 71: 359-362.

13 Schaberg T, Rebhan K, Lode H. Risk factors for side-effects of isoniazid, rifampin and pyrazinamide in patients hospitalized for pulmonary tuberculosis. Eur Respir J 1996; 9: 2026-2030.

14 Ohkawa K, Hashiguchi M, Ohno K, et al. Risk factors for antituberculous chemotherapy-induced hepatotoxicity in Japanese pediatric patients. Clin Pharmacol Ther 2002; 72: 220-226.

15 Yee D, Valiquette C, Pelletier M, Parisien I, Rocher I, Menzies D. Incidence of serious side effects from first-line antituberculosis drugs among patients treated for active tuberculosis. Am J Respir Crit Care Med 2003; 167: 1472-1477.

16 Chang KC, Leung CC, Yew WW, Ho SC, Tam CM. A nested case-control study on treatment-related risk factors for early relapse of tuberculosis. Am J Respir Crit Care Med 2004; 170: 1124-1130.

17 Hong Kong Chest Service/British Medical Research Council. Controlled trial of four thrice-weekly regimens and a daily regimen all given for 6 months for pulmonary tuberculosis. Lancet 1981; 1: 171-174.

18 Hong Kong Chest Service/Tuberculosis Research Centre, Madras/British Medical Research Council. A controlled trial of 3-month, 4-month, and 6-month regimens of chemotherapy for sputum-smear-negative pulmonary tuberculosis. Results at 5 years. Am Rev Respir Dis 1989; 139: 871-876.

19 Jindani A, Nunn AJ, Enarson DA. Two 8-month regimens of chemotherapy for treatment of newly diagnosed pulmonary tuberculosis: international multicentre randomised trial. Lancet 2004; 364: 1244-1251.

20 Zierski M, Bek E. Side-effects of drug regimens used in short-course chemotherapy for pulmonary tuberculosis. A controlled clinical study. Tubercle 1980; 61: 41-49.

21 British Thoracic Association. A controlled trial of six months chemotherapy in pulmonary tuberculosis. First Report: results during chemotherapy. Br J Dis Chest 1981; 75: 141-153.

22 Kopanoff DE, Snider DE, Caras GJ. Isoniazid-related hepatitis: A US Public Health Service cooperative surveillance study. Am Rev Respir Dis 1978; 117: 991-1001. 